\title{
Emergency medical service evaluations for chest pain during the COVID-19 lockdown in Hollands- Midden, the Netherlands
}

Enrico de Koning ( $\nabla$ e.r.de_koning@lumc.nl )

LUMC https://orcid.org/0000-0002-9343-0717

Mark Boogers

LUMC

Jan Bosch

RAVHM

Matthijs de Visser

RAVHM

Martin Schalij

LUMC

Saskia Beeres

LUMC

Research Article

Keywords: COVID-19, chest pain, STEMI, OHCA

Posted Date: October 30th, 2020

DOI: https://doi.org/10.21203/rs.3.rs-100573/v1

License: (1) This work is licensed under a Creative Commons Attribution 4.0 International License.

Read Full License 


\section{Abstract}

Objective: To assess whether the COVID-19 lockdown might have had negative indirect health effects, as people seem to have been reluctant to seek medical care.

Methods: All emergency medical service (EMS) rides for chest pain and out-of-hospital cardiac arrest (OHCA) in the Dutch region Hollands-Midden (population served $>800.000$ ) were evaluated during the initial 6 weeks of the COVID-19 lockdown and compared to the same period in 2019 in two cohorts. The primary end-point was the incidence of evaluated chest pain patients during the COVID-19 lockdown. In addition, the incidence of EMS evaluations of ST elevation myocardial infarction (STEMI) and OHCA were assessed.

Results: During the COVID-19 lockdown period, the EMS evaluated 927 chest pain patients (49\% male, $62 \pm 17$ years) as compared to 1041 patients ( $51 \%$ male, $63 \pm 17$ years) in the same period in 2019 corresponding with a significant relative risk reduction of $0.88(95 \% \mathrm{Cl} 0.81-0.96 ; \mathrm{P}=0.006)$. Similarly, there was a significant reduction in the incidence of STEMI patients (RR 0.52; $P=0.009$ ) whereas the incidence of OHCA (RR 1.23; $\mathrm{P}=0.29$ ) remained unchanged.

Conclusion: During the COVID-19 lockdown, there was a significant decrease in patients with chest pain evaluated by the EMS paralleled by a reduction in STEMIs, while the incidence of OHCA remained similar. While the reason for the decrease in chest pain and STEMI consultations is not entirely clear, more attention should be drawn to the importance of contacting the EMS in case of suspected cardiac symptoms in possible future lockdowns.

\section{What'S New?}

- The COVID-19 lockdown has shown a decrease in STEMI admissions and an increase in OHCA in heavily affected areas worldwide, suggesting a reluctance to contact medical professionals when experiencing chest pain.

- Until now reports have focused on outcomes (in STEMI and OHCA), this is the first report focusing on the amount of EMS evaluations for chest pain. Also this is the first report to focus on chest pain in the Netherlands.

- This report showed a significant decrease in chest pain and STEMI consultations, thus patients experiencing chest pain should be actively encouraged to contact medical professionals during COVID-19 lockdowns.

\section{Introduction}

The rapid spread of the severe acute respiratory syndrome coronavirus 2 (SARS-CoV-2), responsible for coronavirus disease 2019 (COVID-19), urged the Dutch government to announce a national lockdown on March $16^{\text {th }}$ 2020. Apart from social distancing, people were encouraged to stay home as much as 
possible and schools were closed. These measures were effective in controlling the spread of the virus and reduced the pressure on the health care system. There is growing interest in the indirect health effects of the COVID-19 lockdown period. On the one hand, improved air quality and reduced work-related stress might have been beneficial. On the other hand, fear of catching the virus made people cancel, postpone or limit even urgent medical treatments potentially resulting in life-threatening situations [1]. Indeed, during the COVID-19 lockdown period, a significant increase in out-of-hospital cardiac arrests (OHCA) has been observed in Italy and France [2-4]. This finding can be partly attributed to the complications of COVID-19, but may also be related to late presentations of ST elevation myocardial infarction (STEMI) patients with electrical or mechanical complications. Consistently, a decrease in STEMI admissions in the COVID-19 pandemic period has been observed in Italy, France, Spain and the USA [5-11]. Tan et al, analysing cardiac catheterizations in California, reported that the drop in STEMI admissions was also paralleled by a decrease in coronary catheterizations for patients with unstable angina and non-ST elevation myocardial infarction [12]. However, it remains to be determined whether chest pain patients across the board were reluctant to seek acute medical care during the COVID-19 lockdown.

The aim of the current study was to investigate the incidence of chest pain patients evaluated by the Emergency Medical Service (EMS) during the COVID-19 lockdown in the Netherlands. Simultaneously, EMS alerts for STEMI and OHCA were assessed. These data may attribute to the understanding of the indirect health effects of the COVID-19 lockdown and may aid in forthcoming guidelines for acute care during possible future lockdowns.

\section{Methods}

The AmbuSuite database (Topicus, the Netherlands) contains data of all ambulance rides performed by the Regional Ambulance Service Hollands-Midden (RAVHM), the EMS of the Dutch security region Hollands-Midden with over 800.000 inhabitants. All data in the AmbuSuite database are collected prospectively by the paramedics on the ambulance and include patient data (medical history, main complaint, other symptoms, vital parameters, electrocardiogram and working diagnosis) as well as data regarding the ride (dispatch time, time of arrival at the scene, time of arrival at the hospital and address of scene and hospital). The decision to dispatch an ambulance to a patient is made in the regional control centre and based on the Advanced Medical Priority Dispatch System, Professional Quality Assurance which was similar in 2019 and 2020 [13].

All patients for who an ambulance was dispatched by the RAVHM during the initial 6 weeks of the COVID19 lockdown period in the Netherlands (week 12-17 in 2020) were eligible for inclusion in the current study. Inclusion criteria were age above 18 years and an ambulance ride because of the main complaint chest pain or a non-traumatic OHCA. A non-traumatic OHCA was defined as any cardiac arrest after exclusion of cases with obvious accidental causes, irrespective of whether resuscitation was attempted or not. The EMS reports of all OHCA cases were analysed by 2 experienced reviewers (EK and SB) to determine the cause of OHCA. The OHCA were divided in shockable rhythm and non-shockable rhythm on 
arrival by the EMS with the latter category further subdivided in the following causal categories: cardiac aetiology, related to a COVID-19 infection and unknown aetiology. If the paramedics suspected a STEMI, this diagnosis was verified in the central percutaneous coronary intervention centre in the region. For the control group, the same inclusion criteria and definitions were applied to all patients for who an ambulance was dispatched by the RAVHM in week 12-17 in 2019.

Categorical data are presented as numbers with percentages and compared using the chi-square test. Continuous data are presented as mean \pm standard deviation or median with interquartile range and were compared with a one-way ANOVA or Kruskal-Wallis test. The incidence of chest pain, STEMI and OHCA in the COVID-19 lockdown period were compared with the incidence in the same period in 2019. Incidence and relative risk (RR) were estimated with the known regional population in 2019 and in 2020 from Statistics Netherlands (www.CBS.nl) and compared using the chi-square test. The data were analysed using $\mathrm{R}$ version 3.6.2. P-values lower than 0.05 were considered statistically significant. The institutional medical ethical committee approved the study protocol (G20.111) and waived the need for individual informed consent. All data were coded and anonymized.

\section{Results}

During the Dutch COVID-19 lockdown period, the EMS evaluated 927 chest pain patients. In the same period in 2019, the EMS evaluated 1041 patients. As shown in Table 1, patient characteristics did not differ during the COVID-19 lockdown period compared with the same period in 2019 with exception of the time from dispatch to hospital. In particular, gender and age were similar as well as hemodynamic parameters. Although the time from dispatch to patient was similar in both groups, the total time from dispatch to hospital was significantly longer during the COVID-19 lockdown period than during the same period in 2019 (52.0 \pm 15.2 vs. $47.5 \pm 13.9$ minutes; $P<0.001)$.

Table 1. Characteristics of chest pain patients evaluated during the COVID-19 lockdown period and the same period in 2019 . 


\begin{tabular}{|l|c|c|c|}
\hline \multicolumn{3}{|c|}{ COVID-19 lockdown (n=927) } \\
\multicolumn{1}{|l|}{ (n=1041) } \\
\hline Gender (male) & $455(49 \%)$ & $534(51 \%)$ & 0.529 \\
\hline Age (years) & $62 \pm 17$ & $63 \pm 17$ & 0.184 \\
\hline Know coronary disease & $215(23 \%)$ & $224(22 \%)$ & 0.403 \\
\hline Heart rate (bpm) & $86 \pm 28$ & $86 \pm 29$ & 0.415 \\
\hline Systolic blood pressure (mmHg) & $152 \pm 31$ & $150 \pm 31$ & 0.133 \\
\hline Diastolic blood pressure (mmHg) & $88 \pm 19$ & $88 \pm 18$ & 0.487 \\
\hline Time from dispatch to patient (minutes) & $8.0 \pm 3.7$ & $8.1 \pm 3.9$ & 0.536 \\
\hline Time from dispatch to hospital (minutes) & $52.0 \pm 15.2$ & $47.5 \pm 13.9$ & $<0.001$ \\
\hline
\end{tabular}

COVID-19: coronavirus disease 2019

Figure 1. Incidence of chest pain during the COVID-19 lockdown period and the same period in 2019.

As illustrated in Figure 1, the incidence of chest pain, defined as the number of chest pain patients evaluated by the EMS divided by the total amount of inhabitants in the EMS region, was lower during the COVID-19 lockdown period (927/809.104) as compared to the same period in 2019 (1041/802.325). This gives a significant relative risk reduction in the incidence of chest pain in the COVID-19 lockdown period of 0.88 with a $95 \%$ confidence interval $(\mathrm{Cl})$ of $0.81-0.96(P=0.006)$.

Table 2 displays the patients characteristics of STEMI patients in the COVID-19 lockdown period ( $n=23)$ and in the same period in $2019(n=46)$. Of interest, time from dispatch to patient and time from dispatch to hospital were similar in both groups ( $P=0.313$ and $P=0.856$ respectively). The incidence of STEMI, defined as the number of STEMI patients divided by the total amount of inhabitants in the EMS region, was lower during the COVID-19 lockdown period (23/809.104) as compared to the same period in 2019 (46/802.325). Accordingly, during the COVID-19 lockdown period there was a relative risk reduction in STEMI of $0.52(\mathrm{Cl} 0.32-0.85 ; \mathrm{P}=0.009)$ as compared to the same period in 2019 (Figure 2).

Table 2. Characteristics of STEMI patients during the COVID-19 lockdown period and the same period in 2019. 


\begin{tabular}{|l|c|c|c|}
\hline \multicolumn{3}{|c|}{ COVID-19 lockdown (n=23) } \\
\multicolumn{1}{|c|}{$(n=46)$} \\
\hline Gender (male) & $20(87 \%)$ & $39(85 \%)$ & 1 \\
\hline Age (years) & $62 \pm 10$ & $64 \pm 12$ & 0.567 \\
\hline Known coronary disease & $18(78 \%)$ & $41(89 \%)$ & 0.283 \\
\hline Heart rate (bpm) & $76 \pm 38$ & $77 \pm 32$ & 0.945 \\
\hline Systolic blood pressure (mmHg) & $140 \pm 26$ & $138 \pm 46$ & 0.866 \\
\hline Diastolic blood pressure (mmHg) & $86 \pm 20$ & $83 \pm 28$ & 0.614 \\
\hline Time from dispatch to patient (minutes) & $6.6 \pm 2.6$ & $7.5 \pm 4.1$ & 0.313 \\
\hline Time from dispatch to hospital (minutes) & $47.4 \pm 12.8$ & $48.0 \pm 11.1$ & 0.856 \\
\hline
\end{tabular}

COVID-19: coronavirus disease 2019, STEMI: ST-elevation myocardial infarction

Figure 2. Incidence of STEMI during the COVID-19 lockdown period and the same period in 2019.

The patient characteristics of OHCA patients in the COVID-19 lockdown period $(n=56)$ and in the same period in $2019(n=45)$ are shown in Table 3. Both groups were comparable regarding gender, mean age and previously known coronary artery disease. Analysis of the EMS reports of OHCA patients revealed a trend towards an altered cause of OHCA in the COVID-19 lockdown period as compared to the same period in $2019(P=0.05)$. In particular, a shockable rhythm upon arrival by the EMS was found in 15 patients (27\%) in the COVID-19 lockdown period and 18 patients (40\%) in the same period in 2019 and a cardiac aetiology was found in 10 patients (18\%) during the COVID-19 lockdown period and 4 patients (9\%) in the same period in 2019. During the COVID-19 lockdown period, a COVID-19 infection was the probable cause of OHCA in 6 patients $(11 \%)$.

The incidence of OHCA, defined as the number of OHCA patients divided by the total amount of inhabitants in the EMS region, was 56/809.104 during the COVID-19 lockdown period and 45/802.325 in the same period in 2019 (RR 1.23 (Cl 0.83-1.83; $\mathrm{P}=0.29)$, Figure 3).

Table 3. Characteristics of OHCA patients evaluated during the COVID-19 lockdown period and the same period in 2019 . 


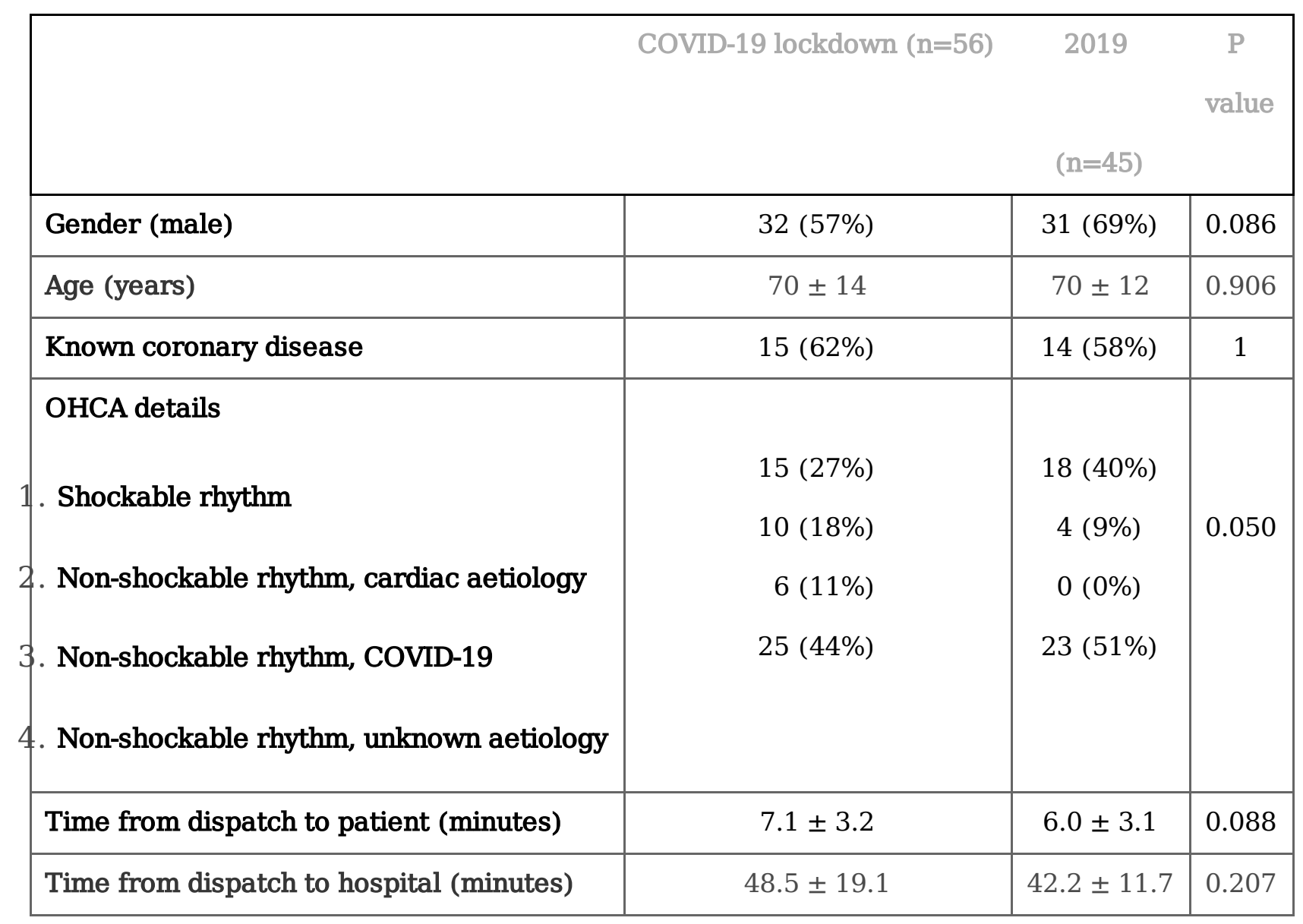

COVID-19: coronavirus disease 2019, OHCA: Out-of-Hospital Cardiac Arrest

Figure 3. Incidence of OHCA during the COVID-19 lockdown period and the same period in 2019.

\section{Discussion}

The main finding of the current study is that the number of patients evaluated by the EMS because of chest pain was lower during the COVID-19 lockdown as compared to the same period in 2019. This was paralleled by a reduction in the incidence of STEMI while the incidence of OHCA remained unchanged.

Beyond the harmful effects of COVID-19 on the respiratory and cardiovascular system [14] there have been major concerns on the indirect negative health effects of the COVID-19 lockdown. Fear of getting infected when alerting the EMS or attending the hospital, made people cancel, postpone or limit medical treatments, potentially resulting in worse outcomes. To better understand the indirect health effects of the COVID-19 pandemic, previous reports have focused on STEMI and OHCAs, whereas so far, it remains unknown whether chest pain patients across its full spectrum were reluctant to request acute medical care during the COVID-19 lockdown. In this perspective, the current study provides novel insights as it showed a significant lower number of chest pain patients evaluated by the EMS during the COVID-19 lockdown as compared to the same period in 2019. The current findings are in line with Tan et al. showing a decline of $26 \%$ in patients with all types of acute coronary syndromes (ACS) undergoing cardiac catheterization in a single centre in California during the COVID-19 pandemic [12]. 
As part of the broad spectrum of chest pain evaluations, the current study also evaluated the EMS alerts for STEMI and showed a significant reduced number of STEMIs during the COVID-19 lockdown when compared to the same period in 2019. This is in line with the previously reported sharp decrease in STEMIs during COVID-19 lockdown [5-11]. De Rosa et al. reported a clear reduction in acute myocardial infarction (AMI) in Italy, where hospitalizations halved compared to the previous year, with a $26.5 \%$ reduction in STEMI diagnoses [5]. Garcia et al. have demonstrated a decrease of $38 \%$ in STEMI referrals of 9 centres in the USA during the COVID-19 period [11].

Our reported decline in chest pain patients as well as STEMIs admitted to hospitals during the COVID-19 lockdown period may conceptually be attributed to different pathophysiologic, environmental and behavioural factors. One can hypothesize that less vigorous physical exertion or reduced physiological stress during a lockdown can result in fewer coronary plaque ruptures [15]. Furthermore, changes in the environment may play a role in a lower incidence of cardiovascular morbidity and mortality, since the COVID-19 lockdown has been associated with a dramatic decline in air pollution $[16,17]$. A reduced exposure to such air pollution due to less traffic and industrial activities during a lockdown, may lead to fewer cardiovascular events. Studies have shown that even short-term exposure to elevated air pollution or traffic exposure was positively associated with elevated risk for myocardial infarctions [18]. Several reports have also shown more hospital admissions for ischemic heart disease with short-term elevation of inhalable or particulate matter air pollution [19]. Last, but most probably not least, behavioural changes may contribute to less EMS evaluations for chest pain or STEMIs, since patients may be reluctant to seek medical contact as they fear being infected or patients are unwilling to burden the health care systems even further.

Previous studies reported an increase in OHCAs during COVID-19 lockdowns [2-4]. Marijon et al suggested that OHCA occurring in patients with respiratory or cardiovascular complications of COVID-19 as well as OHCA related to advanced cardiac injury in late presenters of a STEMI might explain the observed increase in OHCA [4]. In the current study the incidence of OHCA remained unchanged. There was a trend, however, to an altered cause of OHCA with less patients with a shockable rhythm upon arrival by the EMS and more patients with cardiac complaints shortly before the OHCA during the COVID-19 lockdown period. Conceptually, this may concern patients who were reluctant to seek medical help in an earlier phase of an acute myocardial infarction. This, combined with the fact that the total number of OHCA patients in the current study was relatively small and that the number of COVID-19 patients in the Netherlands was relatively low as compared to Italy and France, might explain that in the current study only a shift in causes of OHCA was found rather than an increase in OHCA.

When interpreting the results of the current study, the strengths and limitations should be taken into account. The most important strength is the use of the AmbuSuite database containing prospectively collected data of all ambulance rides in entire security region Hollands-Midden with over 800,000 inhabitants. This database, however, does not provide insight in people with chest pain who decide not to contact the EMS. In addition, it does not contain individual outcome data. Therefore, it was not possible 
to analyse whether clinical outcome of chest pain patients evaluated by the EMS was better or worse during the COVID-19 lockdown period.

To conclude, this study showed a significant decrease in patients with chest pain evaluated by the EMS. This was paralleled by a reduction in the incidence of STEMI, while the incidence of OHCA remained unchanged. While the reason for the decrease in chest pain and STEMI is not entirely clear, there are multiple possible factors. A decrease in physical exertion, a dramatic decrease in air pollution and reluctance to contact medical authorities during the COVID-19 lockdown could have played a role in the reduced number of ambulance rides for chest pain and STEMI. Catching the public's attention to the importance of contacting the EMS in case of suspected cardiac complains may help to reduce the secondary health damage in case of possible future lockdowns.

\section{Declarations}

\section{Acknowledgements}

EK, MB and SB have substantially contributed to conception and design, acquisition of data and analysis and interpretation of data. All authors have drafted or revised the article for important intellectual content and gave final approval for the version to be published. This study complies with the Declaration of Helsinki and was approved by the locally appointed ethics committee.

\section{Sources of funding}

The Department of Cardiology of the Leiden University Medical Center receives unrestricted research and educational grants from Boston Scientific, Medtronic, and Biotronik outside the submitted work.

\section{Conflict of interest}

None declared

\section{References}

1. Rosenbaum, L., The Untold Toll - The Pandemic's Effects on Patients without Covid-19. N Engl J Med, 2020. 382(24): p. 2368-2371.

2. Baldi, E., et al., Out-of-Hospital Cardiac Arrest during the Covid-19 Outbreak in Italy. N Engl J Med, 2020.

3. Baldi, E., et al., COVID-19 kills at home: the close relationship between the epidemic and the increase of out-of-hospital cardiac arrests. Eur Heart J, 2020.

4. Marijon, E.K., N; Jost, D; Perrot, D; Frattini, B; Derkenn, C; Sharifzadehgan, A; Waldmann, V; Beganton, F; Narayanan, K; Lafont, A; Bougouin, W; Jouven, X, Out-of-hospital cardiac arrest during the COVID19 pandemic in Paris, France: a population-based, observational study. Lancet Public Health, 2020. 
5. De Rosa, S., et al., Reduction of hospitalizations for myocardial infarction in Italy in the COVID-19 era. Eur Heart J, 2020. 41(22): p. 2083-2088.

6. De Filippo, 0., et al., Reduced Rate of Hospital Admissions for ACS during Covid-19 Outbreak in Northern Italy. N Engl J Med, 2020.

7. Rangé, G., R. Hakim, and P. Motreff, Where have the STEMIs gone during COVID-19 lockdown? Eur Heart J Qual Care Clin Outcomes, 2020.

8. Rodriguez-Leor, O. and B. Cid-Alvarez, STEMI care during COVID-19: losing sight of the forest for the trees. JACC Case Rep, 2020.

9. Zitelny, E., N. Newman, and D. Zhao, STEMI during the COVID-19 Pandemic - An Evaluation of Incidence. Cardiovasc Pathol, 2020. 48: p. 107232.

10. Solomon, M.D., et al., The Covid-19 Pandemic and the Incidence of Acute Myocardial Infarction. N Engl J Med, 2020.

11. Garcia, S., et al., Reduction in ST-Segment Elevation Cardiac Catheterization Laboratory Activations in the United States during COVID-19 Pandemic. J Am Coll Cardiol, 2020.

12. Tan, W., et al., Single Center Trends in Acute Coronary Syndrome Volume and Outcomes During the COVID-19 Pandemic. Cardiol Res, 2020. 11(4): p. 256-259.

13. Heward, A., M. Damiani, and C. Hartley-Sharpe, Does the use of the Advanced Medical Priority Dispatch System affect cardiac arrest detection? Emergency Medicine Journal, 2004. 21(1): p. 115118.

14. Clerkin, K.J., et al., COVID-19 and Cardiovascular Disease. Circulation, 2020. 141(20): p. 1648-1655.

15. Kato, A., et al., Physical exertion as a trigger of acute coronary syndrome caused by plaque erosion. J Thromb Thrombolysis, 2020. 49(3): p. 377-385.

16. Connerton, P., et al., Air Quality during COVID-19 in Four Megacities: Lessons and Challenges for Public Health. Int J Environ Res Public Health, 2020. 17(14).

17. He, G., Y. Pan, and T. Tanaka, The short-term impacts of COVID-19 lockdown on urban air pollution in China. Nature Sustainability, 2020.

18. Lelieveld, J., et al., Cardiovascular disease burden from ambient air pollution in Europe reassessed using novel hazard ratio functions. Eur Heart J, 2019. 40(20): p. 1590-1596.

19. Brook, R.D., et al., Particulate matter air pollution and cardiovascular disease: An update to the scientific statement from the American Heart Association. Circulation, 2010. 121(21): p. 2331-78.

\section{Figures}




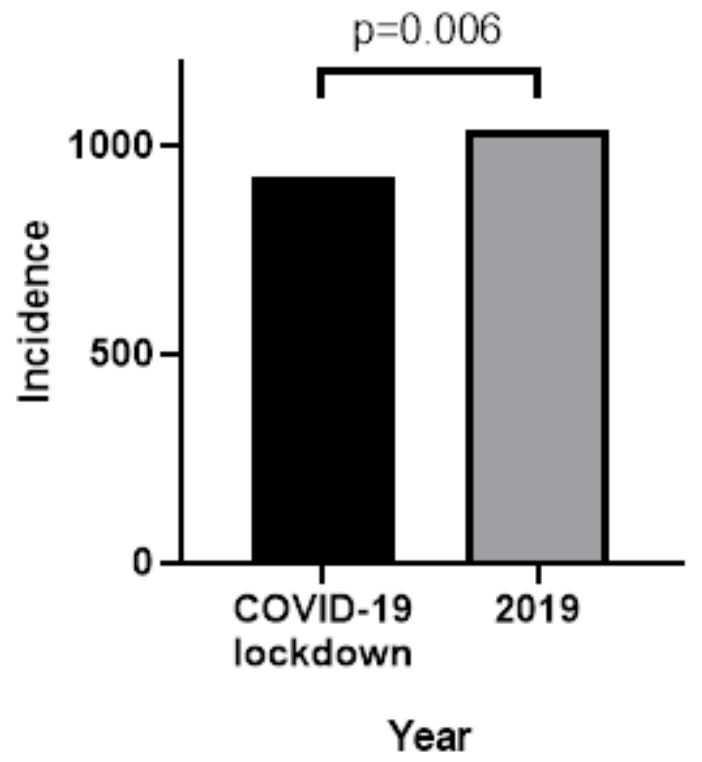

Figure 1

Incidence of chest pain during the COVID-19 lockdown period and the same period in 2019.

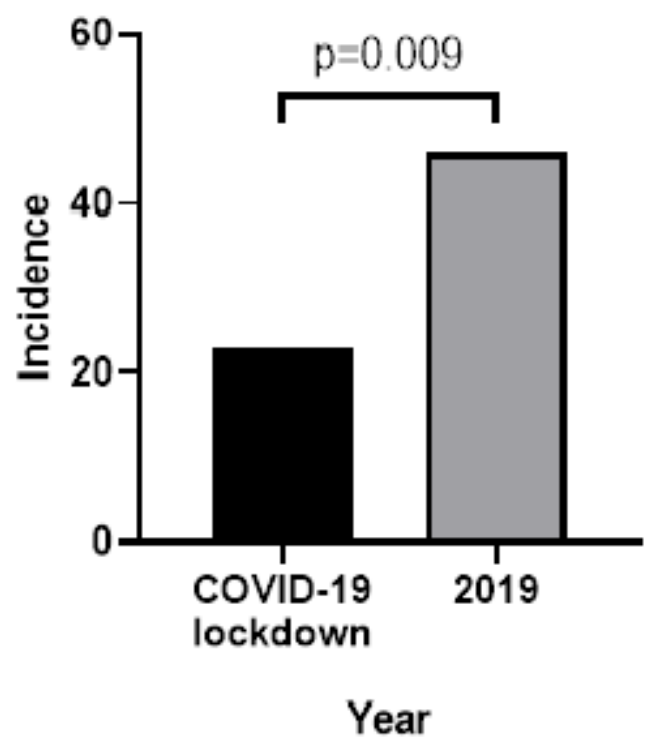

Figure 2

Incidence of STEMI during the COVID-19 lockdown period and the same period in 2019. 


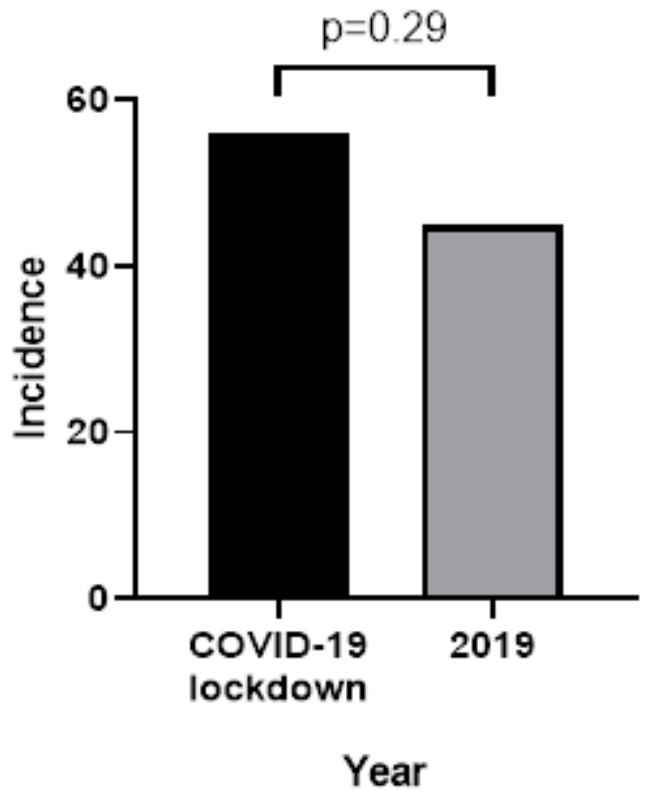

Figure 3

Incidence of OHCA during the COVID-19 lockdown period and the same period in 2019.

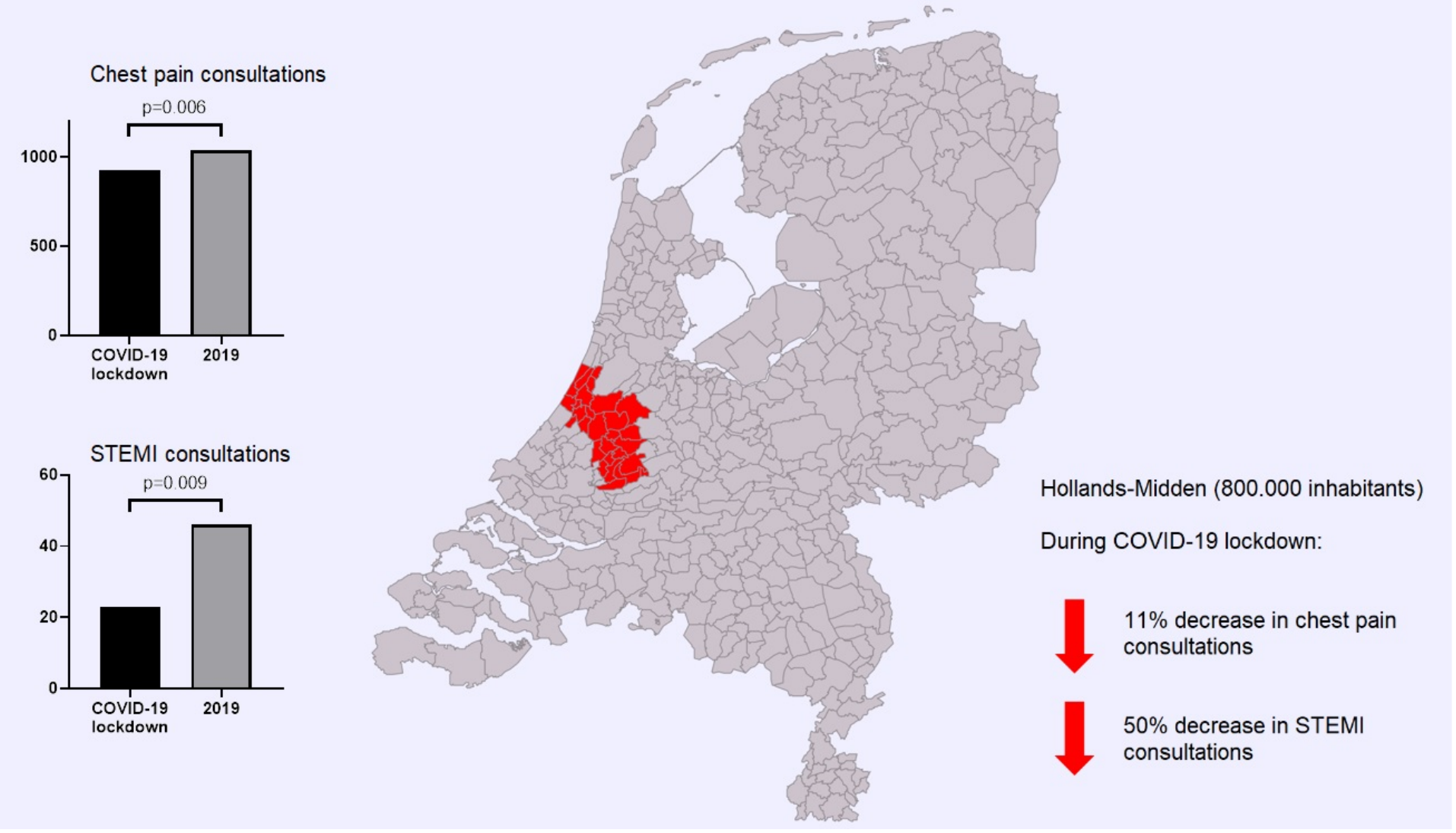

Figure 4

Graphic Abstract 